Available online at www.eccomasproceedia.org

Eccomas Proceedia COMPDYN (2021) 1922-1933

ECCOMAS

Proceedia
COMPDYN 2021

$8^{\text {th }}$ ECCOMAS Thematic Conference on Computational Methods in Structural Dynamics and Earthquake Engineering

M. Papadrakakis, M. Fragiadakis (eds.)

\title{
DISPERSION DESIGN OF 1D PERIODIC DISCRETE SYSTEMS USING LOG-DET HEURISTICS
}

\author{
Anton Tkachuk ${ }^{1}$, and Mykola Tkachuk ${ }^{2}$ \\ ${ }^{1}$ Mechanical and Materials Engineering, Karlstad University \\ 65188 Karlstad, Sweden \\ e-mail: anton.tkachuk@kau.se \\ 2 Department of Theory and Computer-Aided Design of Mechanisms and Machines \\ National Technical University "Kharkiv Polytechnic Institute" \\ Kyrpychova str., 2, Kharkiv, Ukraine, 61002 \\ e-mail: m.tkachuk@tmm-sapr.org
}

\begin{abstract}
A new class of performance functions for dispersion design of $1 D$ periodic discrete systems using matrix rank is considered, and its regularization using existent log-det heuristics is proposed. As an input, the desired dispersion dependency of a branch is used. The design space is defined by a finite set of continuous parameters such as mass or stiffness constants. The mass and stiffness matrices are assumed to depend linearly on these parameters. Ideally, the representative dynamic stiffness matrix is singular at every point of the desired branch. Instead, the sum of ranks for the representative dynamic stiffness matrix evaluated at several discrete points is minimized using a surrogate log-det objective. This approach avoids ordering or tracking of eigenfrequencies and reduces the design problem to a sequence of quadratic programming problems. The considered periodic discrete systems are simplified objects for method development with a future goal of dispersion design of acoustic metamaterials. Also, a combination with reduced-order techniques is conceptually tested.
\end{abstract}

Keywords: Periodic systems, rank minimization heuristics, optimization-based material design, log-det heuristics, reduced-order modeling.

ISSN:2623-3347 (C) 2021 The Authors. Published by Eccomas Proceedia.

Peer-review under responsibility of the organizing committee of COMPDYN 2021.

doi:10.7712/120121.8611.18609 


\section{INTRODUCTION}

Dispersion design in acoustics seeks a material or a system that interacts with acoustic waves in desired manner. Typical examples of desired behavior are prohibiting wave propagation in a specific frequency range via stopping bands in metamaterials and elimination of reflected waves with anechoic tiles. Usually, these designs are realized as an array of repeating heterogeneous cells. Different options are available for manufacturing heterogeneous cells and they can use solid-solid, solid-liquid or solid-air interfaces. Each cell can be described by a large number of design parameters, which makes finding the design a challenging problem.

A common approach is to introduce a performance function that relates the design variables to desired performances and to use it in a gradient-based optimization or integer programming, see examples for maximizing relative band gap in [2] or design of self-collimating materials in [10]. Unfortunately, the common performance functions are implicit functions of dynamic equilibrium equations and their sensitivities are sometimes difficult to obtain, see [13] and references herein. Alternative integer programming approaches need very large number of iterations, e.g. of genetic algorithms. In this contribution a new class of performance functions based on matrix rank is proposed and discussed. An advantage of using matrix rank is that several explicit surrogate functions for rank approximation are available simplifying computation of the performance functions and their sensitivities.

The problem of minimizing the rank of a matrix is known to be computationally hard, see $[12,7.3]$ and it is attributed to be NP-hard in general case [11]. Fortunately, several available heuristics can efficiently solve it. These heuristics rely on different tractable relaxations of the problem, e.g. a nuclear norm minimization, weighted norm minimization or minimization of the logarithm of the determinant $[4,5,9]$. Tractable means here that one quadratic function minimization or SVD per an iteration step is required and the number of such iterations is affordable. Unfortunately, these heuristic methods guarantee the minimum rank solution only in case of well-conditioned equality constraints on the matrix, see the proof in [11]. Various numerical experiments show a satisfactory quality of the solutions obtained by the heuristics for applications as low-rank matrix completion [5, 9], low-order system realization [5] and lowest-dimension embedding of points in Euclidean space [5]. However, these methods are not wide spread for design in vibration or acoustic systems so far. An example in [1] uses rank minimization for reducing dispersion error in spatial discretizations of reciprocal mass matrices. Therefore, exploring rank minimization formulations in dispersion design is promising.

For simplicity, examples here are limited to 1D periodic undamped systems consisting of point masses and springs. Only continuous parameters for mass and stiffness constants are used. Constraints are applied only on upper and lower limits of parameter values.

The contribution is organized as follows. First, equations of motion for a repetitive patch are introduced and a rank minimization objective is formulated in Section 2. A log-det heuristic is applied to the rank minimization problem and two illustrative algebraic examples are discussed in Section 3. A combination of log-det heuristic with the reduced-order techniques is described and illustrated with one algebraic example in Section 4. A numerical example for a uniform back-bone mass-spring chain with a side branch at every second mass element are considered in Section (5). Conclusions are presented in Section 6.

\section{EQUATION OF MOTION AND RANK MINIMIZATION OBJECTIVE}

The equations of motion for an infinite periodic mechanical system can be formulated in different ways, see a comprehensive textbook [3]. Here, a form following [8] is considered for 
$1 \mathrm{D}$ case. The representative patch of the periodic structure is assumed to have $n_{\text {rep }}$ unknown amplitudes denoted as $\tilde{U}$. These amplitudes satisfy equations

$$
\mathbf{Q}\left(\mathbf{K}(\mathbf{s})-\omega^{2} \mathbf{M}(\mathbf{s})\right) \mathbf{S}(k) \tilde{\mathbf{U}}=\mathbf{0},
$$

where $\omega$ and $k$ are the angular frequency and wavenumber, $\mathbf{K}$ and $\mathbf{M}$ are the stiffness and mass matrix. Herein, affine dependence of the stiffness and mass matrix on $n_{s}$ parameters $\mathbf{s}$ is assumed with

$$
\mathbf{K}(\mathbf{s})=\mathbf{K}_{0}+\sum_{j=1}^{n_{s}} \mathbf{K}_{j} s_{j}, \quad \mathbf{M}(\mathbf{s})=\mathbf{M}_{0}+\sum_{j=1}^{n_{s}} \mathbf{M}_{j} s_{j} .
$$

These matrices are assembled for the periodic cell and a layer of neighboring elements. The periodic boundary conditions are enforced via a shift matrix $\mathbf{S}$ whose entries on the slave side are proportional to the phase $\operatorname{shift} \exp (i k \cdot \Delta x)$ along the lattice vector for the periodic cell $\Delta x$. The matrix $Q$ filters degrees of freedom of the representative patch $\tilde{\mathbf{U}}$ from the total model. Thus, the equation of motion as in equation (1) allows us to define the representative dynamic stiffness matrix (RDSM) with

$$
\mathbf{A}(\mathbf{s}, k, \omega)=\mathbf{Q}\left(\mathbf{K}(\mathbf{s})-\omega^{2} \mathbf{M}(\mathbf{s})\right) \mathbf{S}(k) .
$$

We denote with $\mathbf{A}_{j}:=\mathbf{A}\left(\mathbf{s}, k_{j}, \omega_{j}\right)$ the RDSM evaluated at a specific frequency-wavenumber pair $\left(k_{j}, \omega_{j}\right)$. Solvability of the equation of motion (1) at a frequency-wavenumber pair requires that the corresponding RDSM is singular or it has a deficit of rank with

$$
\operatorname{rank} \mathbf{A}_{j}<\operatorname{dim}\left(\mathbf{A}_{j}\right)=n_{\text {rep }}, \quad j=\overline{1, n_{w}},
$$

where $n_{w}$ is the number of sampled frequency-wavenumber pairs. Inspection of all $n_{w}$ inequalities is still a hard problem. Instead, a good solution is sought that minimizes the sum of the ranks of RDSM for all pairs $j$ with respect to parameters s. Such a sum now defines the objective of the dispersion design. This sum is equal to the product $\left(n_{\text {rep }} n_{w}\right)$ at an initial value of parameters $\mathbf{s}_{0}$. During the optimization process, for certain values of the parameters $\mathbf{s}_{k}$ the rank of some of the matrices $\mathbf{K}_{\text {dyn,rep }}\left(\mathbf{s}_{k}, \omega_{j}, \mathbf{k}_{j}\right)$ reduces to $\left(n_{\text {rep }}-1\right)$ meaning that exactly one branch of the dispersion relation crosses the prescribed frequency-wavevector pair $\left(\omega_{j}, \mathbf{k}_{j}\right)$. Reduction of the rank to $\left(n_{\text {rep }}-2\right)$ or less would require that more than one branch of the dispersion relation crosses the prescribed frequency-wavevector pair, i.e. two or more waves can propagate with the same wavenumber and frequency, but a different mode. Such a multiple crossing is possible but it does not significantly influence the design for a sufficiently large number of target pairs per branch. Therefore, reduction of the objective increases the number of the frequency-wavevector pairs at which the designed material follows the wanted dispersion behavior. Thus, this objective can estimate the quality of the design.

To bring the objective to a concise form, let us define the rank identity for a block diagonal matrix with

$$
\sum_{j=1}^{n_{w}} \operatorname{rank} \mathbf{A}_{j}=\operatorname{rank}_{\operatorname{diag}}=\overline{1, n_{w}}\left(\mathbf{A}_{j}\right)
$$

is valid for any set of square matrices $\mathbf{A}_{j}$. Here, an operator diag denotes a constructor of a block diagonal matrix from a list of rectangular matrices. Finally, the objective reads

$$
J(\mathbf{s})=\operatorname{rank} \operatorname{diag}_{j=1, n_{w}}\left(\mathbf{A}_{j}\right) .
$$


The latter problem is still an affine rank minimization problem. Affinity easily follows from the definition of individual subbocks of the objective matrix. Recasting leads to

$$
\begin{aligned}
& \operatorname{diag}_{j=1, n_{w}}\left(\mathbf{A}_{j}\right)=\operatorname{diag}_{j=\overline{1, n_{w}}}\left(\mathbf{Q}\left(\mathbf{K}(\mathbf{s})-\omega_{j}^{2} \mathbf{M}(\mathbf{s})\right) \mathbf{S}\left(k_{j}\right)\right) \\
= & \operatorname{diag}_{j=\overline{1, n_{w}}}\left(\mathbf{Q}\left(\mathbf{K}_{0}+\sum_{l=1}^{n_{s}} \mathbf{K}_{l} s_{l}-\omega_{j}^{2}\left[\mathbf{M}_{0}+\sum_{l=1}^{n_{s}} \mathbf{M}_{l} s_{l}\right]\right) \mathbf{S}\left(k_{j}\right)\right)=\mathbf{B}_{0}+\sum_{l=1}^{n_{s}} \mathbf{B}_{l} s_{l},
\end{aligned}
$$

where matrices $\left\{\mathbf{B}_{l}\right\}_{0}^{n_{s}}$ are defined

$$
\mathbf{B}_{l}=\operatorname{diag}_{j=\overline{1, n_{w}}}\left(\mathbf{Q}\left(\mathbf{K}_{l}-\omega_{j}^{2} \mathbf{M}_{l}\right) \mathbf{S}\left(k_{j}\right)\right) .
$$

\section{HEURISTIC RANK MINIMIZATION}

Herein, a focus on log-det heuristics is made because of its simplicity and robustness. The rank minimization problem stated above is solved approximately by log-det heuristics from [5]. A substantial difference to the original method is due to complex entries of the RDSM matrices. These complex entries originate from the shift matrix $\mathbf{S}$ even for the case without damping. The original rank minimization algorithm is valid for real semi-definite matrices. To bring our problem to an appropriated shape, a matrix $\mathbf{X}$ is introduced with

$$
\mathbf{X}=\operatorname{diag}_{j=1, n_{w}}\left(\mathbf{A}^{\mathrm{H}}\left(\mathbf{s}, \omega_{j}, k_{j}\right) \mathbf{A}\left(\mathbf{s}, \omega_{j}, k_{j}\right)\right)
$$

where superscript $\square^{\mathrm{H}}$ denotes Hermitian transpose (the conjugate transpose). The resultant matrix is a Hermitian positive semi-definite matrix, which is sufficient for the original log-det heuristics. The rank of matrix $\mathrm{X}$ coincides with the objective function (6), but dependency on the free parameters is quadratic. A similar trick is used in [1] for dispersion error minimization on a regular spatial grid of finite elements. Now, a rank minimization problem (RMP) is formulated for matrix $\mathbf{X}$ with

$$
\mathbf{s}^{*}=\underset{\mathbf{s} \in \mathcal{K}}{\arg \min }(\operatorname{rank}(\mathbf{X}))
$$

where $\mathcal{K}$ denotes an admissible convex set for parameters s. Rather than solving a RMP, a log-det smooth surrogate for $\operatorname{rank}(\mathbf{X})$ is used

$$
\mathbf{s}^{*}=\underset{\mathbf{s} \in \mathcal{K}}{\arg \min }(\log \operatorname{det}(\mathbf{X}+\delta \mathbf{I})),
$$

where $\delta>0$ is a small regularization constant and $\mathbf{I}$ is as the identity matrix. Despite complex entries of the matrix $\mathbf{X}$, the determinant of a Hermitian positive definite matrix $(\mathbf{X}+\delta \mathbf{I})$ is real and positive. Therefore, the objective function above is real and the optimization problem well defined. Here, we follow the paper [5] and reduce the optimization to a series of quadratic programming problems. First, a Taylor expansion of the objective function in the vicinity of the current value $\mathbf{X}_{k}$ is carried out

$$
\log \operatorname{det}(\mathbf{X}+\delta \mathbf{I}) \approx \log \operatorname{det}\left(\mathbf{X}_{k}+\delta \mathbf{I}\right)+\operatorname{trace}\left(\mathbf{W}_{k}\left(\mathbf{X}-\mathbf{X}_{k}\right)\right),
$$

where $\mathbf{W}_{k}=\left(\mathbf{X}_{k}+\delta \mathbf{I}\right)^{-1}$ denotes the weighting matrix. The weighting matrix is evaluated numerically. Thus, the linearization of the surrogate function (12) has only a dependence on design parameters in the second factor $\left(\mathbf{X}-\mathbf{X}_{k}\right)$. The algorithm is illustrated in Box 1. The linearization of objective leads to real-valued Hessian $H_{j l}$ and real-valued linear part $f_{j}$ with

$$
H_{j l}=\operatorname{Re}\left[\operatorname{trace}\left(\mathbf{W}_{k}\left(\mathbf{B}_{j}^{H} \mathbf{B}_{l}\right)\right)\right] \quad f_{j}=\operatorname{Re}\left[\operatorname{trace}\left(\mathbf{W}_{k}\left(\mathbf{B}_{j}^{H} \mathbf{B}_{0}\right)\right)\right] .
$$




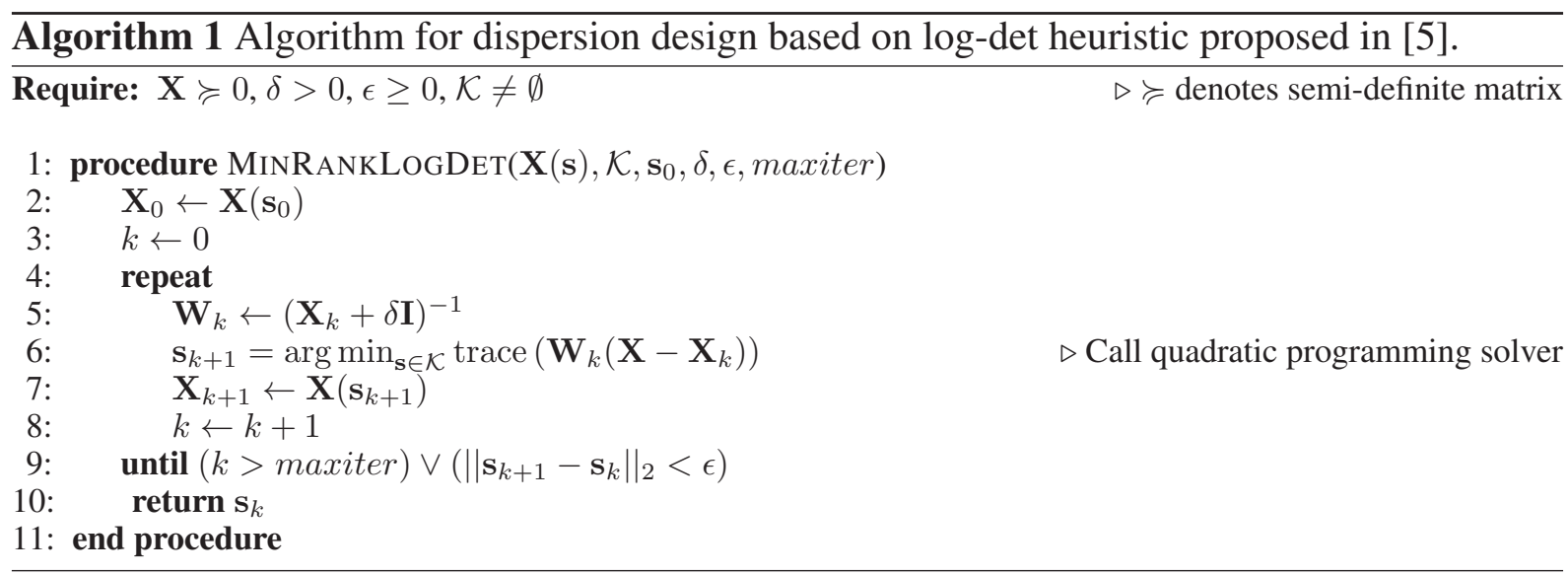

Computation with double precision leads to tiny imaginary parts in the coefficients. Therefore, taking the real part is necessary.

The presented above algorithm is illustrated now with two algebraic examples. Both example use double precision arithmetic and a value of the regularization parameter $\delta=10^{-3}$.

Algebraic example 1. First, a real-valued example is considered, where Hermitian transpose and usual transpose coincide. Find values of two parameters $\mathbf{s}=\left[s_{1}, s_{2}\right]$ within an admissible set $\mathcal{K}=[0,1] \times[0,1]$ that minimize the rank of a square matrix $\mathbf{X} \in \mathbb{R}^{4 \times 4}$ given as

$$
\mathbf{X}=\mathbf{A}^{\mathrm{T}} \mathbf{A}, \quad \mathbf{A}=\mathbf{B}_{0}+s_{1} \mathbf{B}_{1}+s_{2} \mathbf{B}_{2}
$$

with affine representation of matrix $\mathbf{A}$ with matrices $\mathbf{B}_{i}$ defined as

$$
\mathbf{B}_{0}=\left[\begin{array}{cccc}
5000 & 20 & 1 / 3 & 1 / 3 \\
20 & 5 / 4 & 1 / 3 & 1 / 4 \\
1 / 3 & 1 / 3 & 1 / 3 & 1 / 3 \\
1 / 3 & 1 / 4 & 1 / 3 & 1 / 4
\end{array}\right] \quad \mathbf{B}_{2}=\left[\begin{array}{cccc}
-1 & -1 & -1 & -1 \\
-1 & 0 & -1 & 0 \\
-1 & -1 & -1 & -1 \\
-1 & 0 & -1 & 0
\end{array}\right] \quad \mathbf{B}_{3}=\left[\begin{array}{cccc}
0 & 0 & 0 & 0 \\
0 & -2 & 0 & -2 \\
0 & 0 & 0 & 0 \\
0 & -2 & 0 & -2
\end{array}\right] .
$$

This RMP has the global minimum

$$
\mathbf{A}\left(s_{1}=\frac{1}{3}, s_{2}=\frac{1}{8}\right)=\left[\begin{array}{cccc}
\frac{224973482}{9} & \frac{885118}{9} & 0 & 0 \\
\frac{885118}{9} & \frac{3490}{9} & 0 & 0 \\
0 & 0 & 0 & 0 \\
0 & 0 & 0 & 0
\end{array}\right] \Rightarrow \operatorname{rank}(\mathbf{X})=\operatorname{rank}\left(\mathbf{A}^{\mathrm{T}} \mathbf{A}\right)=2
$$

The log-det surrogate function shown in Figure 1 has its global minimum very close to the global minimum of the original problem. The global minimum is observed at the cross-section of two valleys where deficit of rank is one. Decreasing the regularization parameter would make the valley flanks steeper but it does not change qualitatively the shape of the objective function. The evolution of the surrogate objective over iterations is also shown in Figure 1. Convergence 

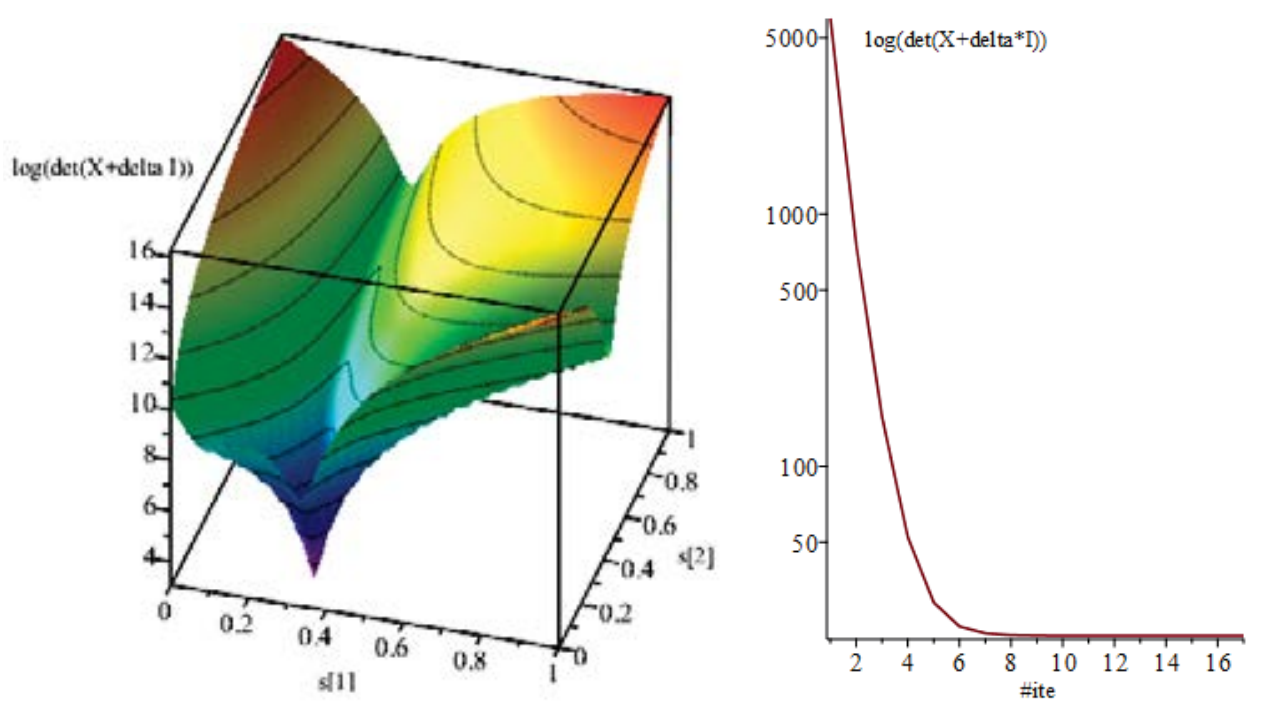

Figure 1: Surrogate objective function $\log \operatorname{det}(\mathbf{X}+\delta \mathbf{I})$ for the algebraic example with a regularization $\delta=10^{-3}$ (left). Convergence of the surrogate objective (right) from starting value $[1 / 6,1 / 12]$.

is achieved from starting value $[1 / 6,1 / 12]$ to the rank- 2 solution

$$
\mathbf{X}^{*}\left(s_{1}=0.333328, s_{2}=0.125539\right) \approx\left[\begin{array}{cccc}
2.5 e 7 & 9.83 e 4 & 0.0264 & 0.00515 \\
9.83 e 4 & 388.0 & 1.09 e-4 & -9.72 e-4 \\
0.0264 & 1.09 e-4 & 1.11 e-9 & -1.13 e-8 \\
0.00515 & -9.72 e-4 & -1.13 e-8 & 2.32 e-6
\end{array}\right]
$$

with two lowest eigenvalues below $10^{-5}$ within ten iterations.

Algebraic example 2. The second algebraic example treats a complex-valued square matrix $\mathbf{X} \in \mathbb{C}^{3 \times 3}$ depending on two parameters $\mathbf{S}=\left[s_{1}, s_{2}\right]$ within an admissible set $\mathcal{K}=[0,1] \times[0,1]$. Hermitian semi-positive matrix $\mathbf{X}$ is defined as

$$
\mathbf{X}=\mathbf{A}^{\mathrm{H}} \mathbf{A}, \quad \mathbf{A}=\mathbf{B}_{0}+s_{1} \mathbf{B}_{1}+s_{2} \mathbf{B}_{2}
$$

with matrices $\mathbf{B}_{i}$ defined

$$
\begin{aligned}
& \mathbf{B}_{0}=\left[\begin{array}{ccc}
5 / 4 & 1 / 3 & -1 / 12+i / 3 \\
1 / 3 & 1 / 3 & i / 3 \\
-1 / 12+i / 3 & i / 3 & -\frac{5}{12}
\end{array}\right] \\
& \mathbf{B}_{2}=\left[\begin{array}{ccc}
0 & -1 & 1-i \\
-1 & -1 & -i \\
1-i & -i & 2
\end{array}\right] \quad \mathbf{B}_{3}=\left[\begin{array}{ccc}
0 & -1 & 1-i \\
-1 & -1 & -i \\
1-i & -i & 2
\end{array}\right]
\end{aligned}
$$

This RMP has the global minimum

$$
\mathbf{A}\left(s_{1}=\frac{1}{3}, s_{2}=\frac{1}{8}\right)=\left[\begin{array}{lll}
1 & 0 & 0 \\
0 & 0 & 0 \\
0 & 0 & 0
\end{array}\right] \quad \Rightarrow \quad \operatorname{rank}(\mathbf{X})=\operatorname{rank}\left(\mathbf{A}^{\mathrm{H}} \mathbf{A}\right)=1
$$


Log-det heuristic approximates this solution within ten iterations $s_{1}=0.334057, s_{2}=0.126277$ from starting value $[1 / 6,1 / 12]$

$$
\mathbf{X}^{*}=\left[\begin{array}{ccc}
0.995 & -0.000721+0.00000133 i & -0.00182-0.000722 i \\
-0.000721-0.00000133 i & 0.00000157 & (1.33+0.247 i) e-6 \\
-0.00182+0.000722 i & (1.33-0.247 i) e-6 & 5.62 e-6
\end{array}\right] .
$$

and it demonstrates work of the log-det heuristic for a Hermitian semi-positive matrix.

\section{COMBINATION WITH REDUCED-ORDER TECHNIQUES}

Increasing size of the representative patch increases rapidly cost of evaluation of the coefficient of quadratic programming problem in (13). Therefore, reducing dimension of the final RMP is very important. Here, a naive projection of the problem onto a reduced basis $\Phi \in \mathbb{C}^{n_{\text {rep }} n_{w} \times n_{r}}, n_{r} \ll n_{\text {rep }} n_{w}$ is proposed in form

$$
\bar{J}(\mathbf{s})=\operatorname{rank}\left(\boldsymbol{\Phi}^{\mathrm{H}} \mathbf{X}(\mathbf{s}) \boldsymbol{\Phi}\right) .
$$

Accuracy and efficiency of the reduced problem depends strongly on the reduced basis. Ideally, it should contain the kernel of the matrix $\mathbf{X}$ at the solution point $\mathbf{s}^{*}$ and have comparable dimension. Practically, using few consequent eigenmodes of $\mathbf{A}$ at the initial approximation $\mathbf{s}_{0}$ is possible and used herein. Formally, this reduce basis can be written in block-diagonal format with

$$
\boldsymbol{\Phi}=\operatorname{diag}_{j=1, n_{w}}\left(\left[\operatorname{eig}\left(\mathbf{A}_{j}\left(\mathbf{s}_{0}\right)\right)\right]_{: n_{l} . . n_{u}}\right),
$$

where eig is an operator returning in columns eigenmodes in ascending order w.r.t. real part and an operation $\square_{:, n_{l} . . n_{u}}$ returns columns $\overline{n_{l}, n_{u}}$ of a matrix. This construction of the reduced basis yields the reduced dimension

$$
n_{r}=n_{w}\left(n_{u}-n_{l}+1\right)
$$

The surrogate log-det function for the rank is computed in the same manner as for the full problem. Finally, a following minimization problem is solved

$$
\mathbf{s}^{*}=\underset{\mathbf{s} \in \mathcal{K}}{\arg \min }\left(\log \operatorname{det}\left(\boldsymbol{\Phi}^{\mathrm{H}} \mathbf{X}(\mathbf{s}) \mathbf{\Phi}+\delta \mathbf{I}\right)\right),
$$

where the dimension of identity matrix is adjusted to $n_{r}$.

We expect that the influence of reduced-order technique is two-fold. First, the dimension of the problem significantly reduces. Second, the conditioning of the matrix $\Phi^{\mathrm{H}} \mathbf{X}(\mathbf{s}) \Phi$ is better than for full matrix $\mathbf{X}(\mathbf{s})$, which helps log-det heuristic to converge faster and allows lower values of the regularization parameter $\delta$. However, checking these two hypothesis goes beyond the contribution.

Algebraic example 3. This example uses setup of algebraic example 1. The influence of the reduced basis on the quality of the solution is studied in for two reduced bases

$$
\boldsymbol{\Phi}_{1}=\left[\begin{array}{cc}
0 & 0 \\
0 & 1 / 5 \\
1 & -1 \\
1 & 1
\end{array}\right], \quad \boldsymbol{\Phi}_{2}=\left[\begin{array}{cc}
1 & 0 \\
0 & 1 \\
-1 & 0 \\
0 & -1
\end{array}\right]
$$


The former basis is very close to the kernel of the matrix $\mathrm{X}$ at the solution point

$$
\operatorname{ker}(\mathbf{X})=\left[\begin{array}{cc}
0 & 0 \\
0 & 0 \\
1 & -1 \\
1 & 1
\end{array}\right]
$$

The surrogate objective function for the reduced basis $\boldsymbol{\Phi}_{1}$ is shown in Figure 2 (left). It has a global minimum close the exact one. The surrogate objective function for the reduced basis $\Phi_{2}$ does not depend on the parameters and it cannot be used. It is an example of extremely unfavorable choice of the reduced basis. Convergence to a solution $s_{1}=0.333337, s_{2}=$ 0.125002 is obtained within 10 iterations. Evolution of the objective during iteration process is shown in Figure 2 (right). The absolute values of objective for the reduced problem are different the full one, which is also clear from comparison of Figure 2 and 1.
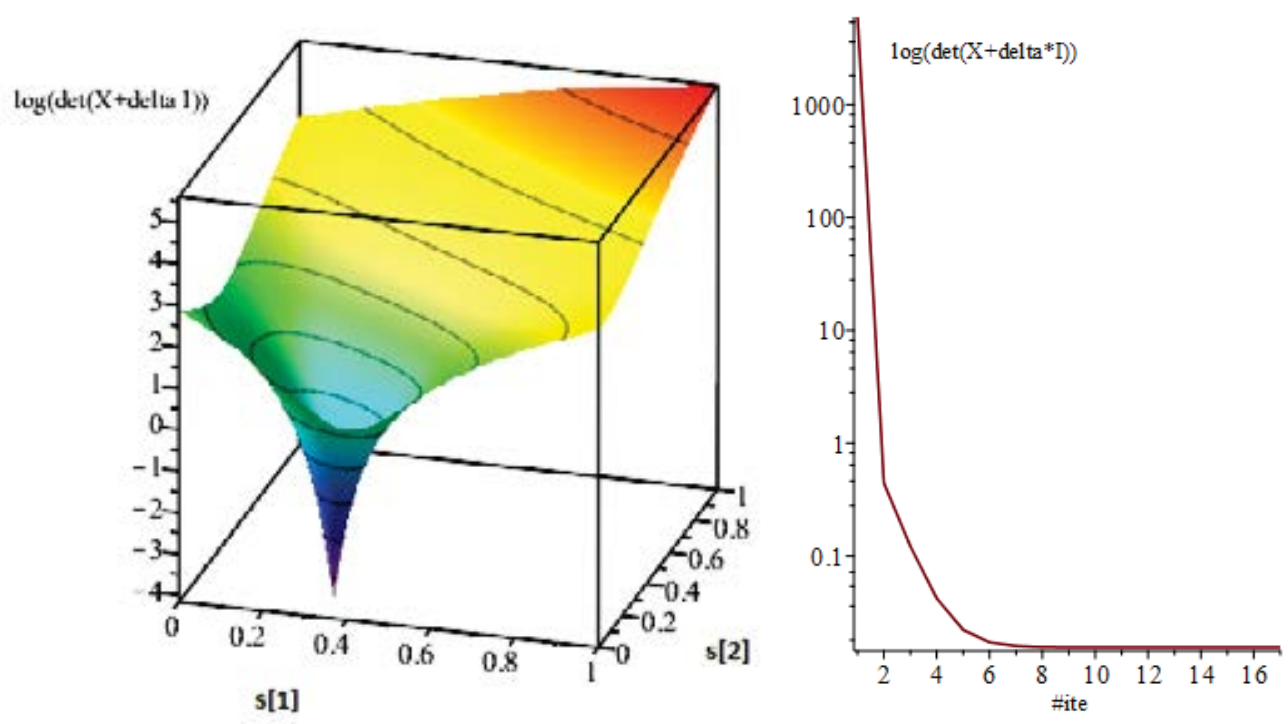

Figure 2: Surrogate objective function $\log \operatorname{det}\left(\boldsymbol{\Phi}^{\mathrm{H}} \mathbf{X}(\mathbf{s}) \boldsymbol{\Phi}+\delta \mathbf{I}\right)$ for the algebraic example with a regularization $\delta=10^{-3}$ using the reduced basis $\boldsymbol{\Phi}_{1}$ (left). Convergence of the reduced surrogate objective (right) from starting value $[1 / 6,1 / 12]$.

\section{NUMERICAL EXAMPLE}

A mechanical example illustrates performance of the proposed approach. A periodic system with a uniform back-bone mass-spring chain and a side branch at every second mass element is shown in Figure 3. Dimension of the representative patch is five, while seven continuous parameters define system properties. The mass and stiffness constants in the back-bone are fixed. Three mass and four stiffness parameters of the side branch can be varied within ranges given in Figure 3. Stiffness parameters are denoted with letter $\beta$ to avoid any confusion with the wavenumber denoted with letter $k$.

The aim is to obtain a dispersion design with an almost constant frequency branch, which also provides a near to zero group velocity. Five target wavenumber-frequency pairs are used 

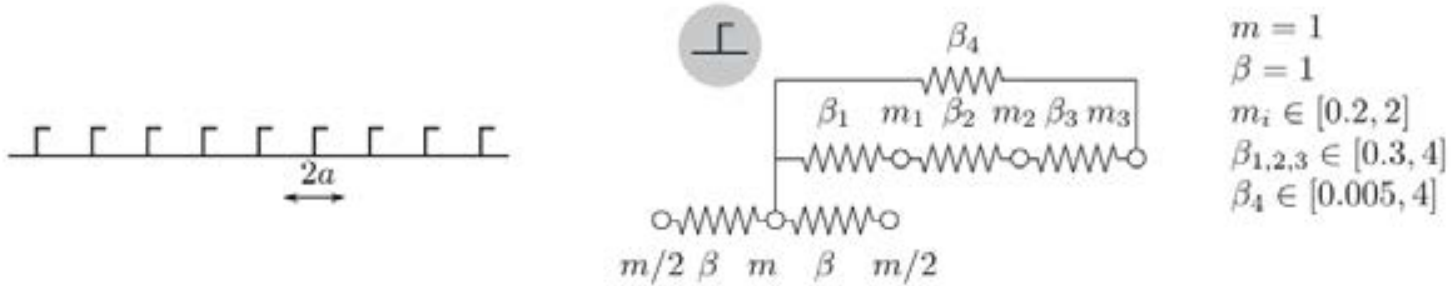

Figure 3: Setup for a periodic discrete system. Overall view on 1D linear mass-spring system with side branch (left) and a zoom in a repetitive structure with topology, parameters and their ranges (right).

with

$$
n_{w}=5, \quad a k_{j}=\frac{\pi j}{n_{w}+1}, \quad \omega_{j}=1.58, \quad j=\overline{1, n_{w}},
$$

where $2 a$ is the physical length of repetitive structure, as shown in Figure 3. At these pairs, the RDSM should be singular and it is achieved by minimizing the rank of the corresponding matrix via log-det heuristic. Three starting value sets for design parameters are listed in Table 1 . The regularization parameter $\delta=3 \cdot 10^{-8}$, convergence tolerance $\epsilon=10^{-10}$ and maximum 15 iterations is used in the log-det heuristic.

A solution is obtained after 15 iterations and the final values are given in Table 1. Reduction of the surrogate objective over iterations is given in Figure 4. These curves have very steep initial phase. Eventually, they flatten and come to saturation. If the convergence tolerance $\epsilon$ for the increment of parameter vector is not reached then the algorithm iterates until the maximum iteration number is reached.

\begin{tabular}{|l|l|l|l|l|l|l|l|}
\hline Case & $\beta_{1}$ & $\beta_{2}$ & $\beta_{3}$ & $\beta_{4}$ & $m_{1}$ & $m_{2}$ & $m_{3}$ \\
\hline Starting values, set I & 1.000 & 1.300 & .6000 & .1000 & 1.000 & 2.000 & 1.200 \\
\hline Starting values, set II & 1.000 & .5000 & .6000 & .1000 & .5000 & 1.000 & 1.200 \\
\hline Starting values, set III & .3000 & .5000 & .6000 & .1000 & .5000 & 2.000 & 2.000 \\
\hline Result, set I & 1.098 & .3000 & .3009 & .3245 & .4443 & .5395 & .2848 \\
\hline Result, set II & 1.089 & .3000 & .3001 & .2883 & .4359 & .5731 & .2676 \\
\hline Result, set III & .3000 & .3000 & .3000 & .0009 & .2681 & .8135 & .2009 \\
\hline Result for ROM, set I & .3558 & .3000 & .3000 & .2932 & .2000 & .3950 & .2759 \\
\hline Result for ROM, set II & .3752 & .3000 & .3000 & .2735 & .2000 & .2700 & .2912 \\
\hline Result for ROM, set III & .3442 & .3153 & .3000 & .2483 & .2000 & .3209 & .2646 \\
\hline
\end{tabular}

Table 1: Starting and final values for dispersion design parameters of the periodic discrete systems.

Figure 5 illustrates the dispersion behavior for the obtained system for starting set I and III. Result obtained with set II is very similar to one obtained for set I. The obtained system satisfies the desired property of having a constant frequency branch for initial set I and II. Notably, this branch is crossed by another branch and it is formed by third frequency for wavenumbers $a k<2.02$ and fourth frequency otherwise. Information about the order of frequencies was not specified in the algorithm. Such a behavior illustrates the advantage of the proposed approach over standard methods, where the objective is formulated with respect to a specific frequency. Solution obtained from the starting set III is different and it satisfies the rank deficit condition only at four out of five points. 

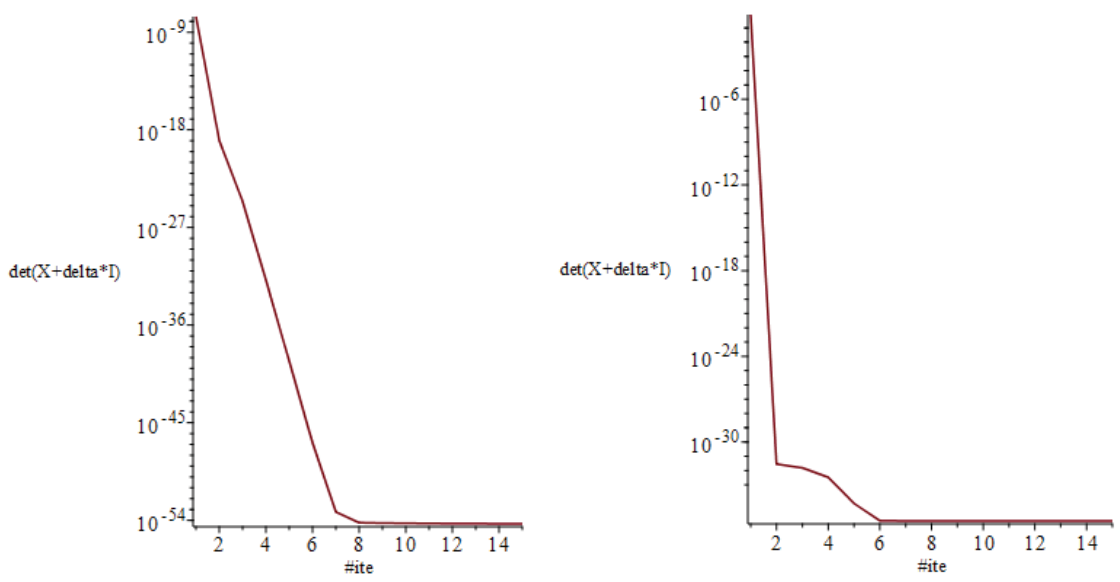

Figure 4: Convergence of the surrogate objective for initial parameter set I (left) and set III (right).
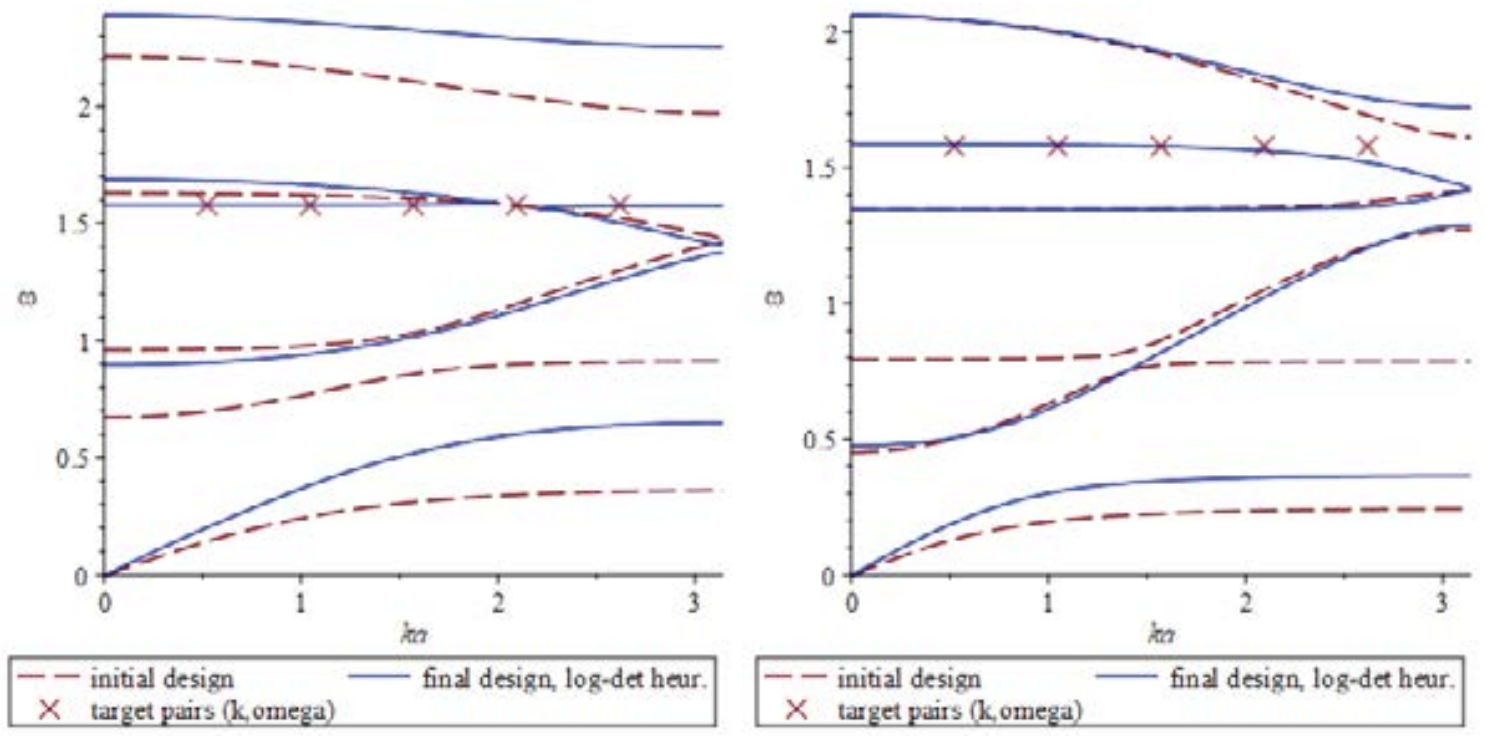

Figure 5: Dispersion behavior of the designed periodic discrete system with prescribed constant frequency $\omega=$ 1.58. Initial and final dispersion behavior for initial parameter set I (left) and set III (right).

Reduced-order technique presented in Section 4 is now applied to dispersion design problem. Dimension of the original system is $n_{w} n_{\text {rep }}=25$. Here, three eigenmodes in span $n_{l}=2$ and $n_{u}=4$ per sampling pair is used. Thus, the problem is reduced to the size $n_{w}\left(n_{u}-n_{l}+1\right)=15$ as explained in equation (25). This reduction is not drastic and it only served to check algebraic correctness of the approach.

Similar results are obtained for three starting parameter sets, see Table 1 and Figure 6. For all initial sets, third branch branch crosses all target pairs and these solutions are considered as satisfactory.

The considered example is undetermined, i.e. it has seven parameters and five conditions to be fulfilled. It is not a surprise that more than one solution is inside the admissible set $\mathcal{K}$ and they are obtained with the proposed approach. It is also clear that the influence of starting approximation and algorithm setting is significant. 

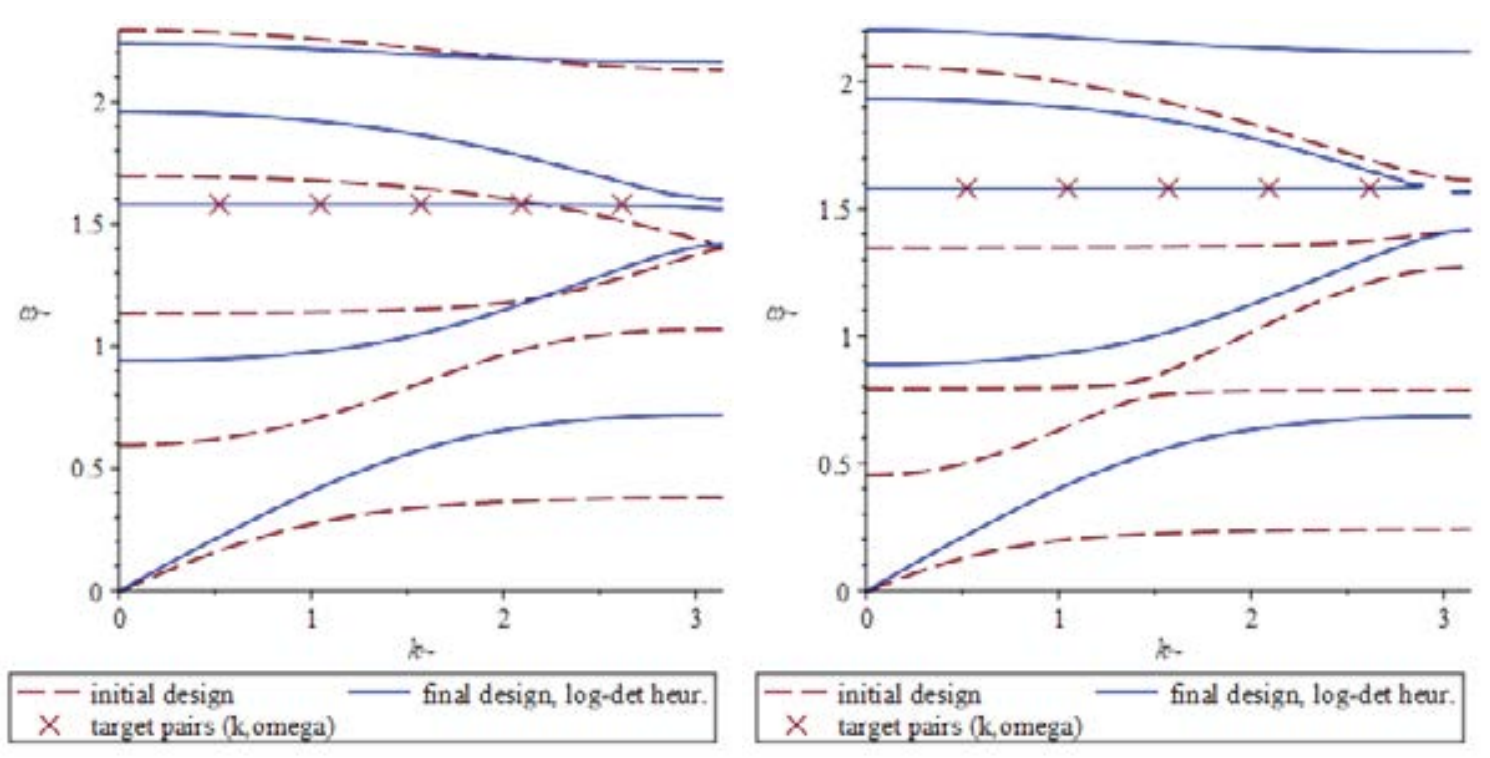

Figure 6: Dispersion behavior of the designed periodic discrete system with prescribed constant frequency $\omega=$ 1.58. Initial and final dispersion behavior for initial parameter set II (left) and set III (right) obtained with reducedorder technique.

\section{CONCLUSIONS}

This contribution considers a special problem of design of 1D periodic discrete systems whose dispersion branch matches a prescribed dependency. For this problem, a new class of performance functions is proposed that explicitly depends on the design variables and does not require tracking of eigenmodes. This performance function is derived in several steps. First, a match to a dispersion branch is collocated in few discrete wavenumber-frequency pairs. Second, this match at discrete pairs is understood as the deficit of the representative dynamic stiffness matrix's rank. Thus, sum of these ranks is identified as a possible performance function and its reduction means quality improvement of the design. Third, the latter rank minimization problem is solved using log-det heuristics. Here, a modification for a case of square complexvalued matrices is proposed. Fourth, the log-det heuristics reduces the problem to a series of quadratic programming problems. The coefficients of the objective quadratic function are obtained here for the case of affine dependency of the mass and stiffness matrices on the free parameters.

Combining the proposed approach with reduced-order techniques is shortly discussed and a version of the reduced basis is proposed.

This approach is illustrated with several algebraic examples and one mechanical example. First algebraic example demonstrates the basic features of the rank minimization using log-det heuristic. Second algebraic example confirms the extension of the log-det heuristic for Hermitian semi-positive matrices. Third algebraic example demonstrates validity of projection-based reduction for rank minimization problems. Finally, a mechanical periodic system is considered and the concept of the dispersion design using log-det heuristic is demonstrated.

Further efforts may be focused on extending the approach to damped systems (achieving so-called metadamping [7]) and exploring the method for larger or multidimensional systems. Also, finding further explicit performance functions for different types of target dispersion behavior is of high scientific interest. 


\section{ACKNOWLEDGMENT}

The work of Anton Tkachuk was supported by the Deutsche Forschungsgemeinschaft (DFG

- German Research Foundation) Projektnummer 442679063 (TK 63/3-1).

\section{REFERENCES}

[1] A. Tkachuk. Customization of reciprocal mass matrices via log-det heuristic. IJNME, 2020, volume 121, pages 690-711.

[2] A. Bacigalupo, G. Gnecco, M. Lepidi, and L. Gambarotta. Machine-learning techniques for the optimal design of acoustic metamaterials. Journal of Optimization Theory and Applications, 2019, 1-24.

[3] P. A. Deymier. Acoustic metamaterials and phononic crystals. Springer Science \& Business Media, 2013.

[4] M. Fazel, H. Hindi, and S. Boyd. A rank minimization heuristic with application to minimum order system approximation. 6:4734-4739, 2001.

[5] M. Fazel, H. Hindi, and S. P. Boyd. Log-det heuristic for matrix rank minimization with applications to hankel and euclidean distance matrices. In Proceedings of the 2003 American Control Conference, 2003, volume 3, pages 2156-2162. IEEE, 2003.

[6] M. J. Frazier and M. I. Hussein. Viscous-to-viscoelastic transition in phononic crystal and metamaterial band structures. JASA, 138(5):3169-3180, 2015.

[7] M. I. Hussein and M. J. Frazier. Metadamping: An emergent phenomenon in dissipative metamaterials. JSV, 332(20):4767-4774, 2013.

[8] D. Mead. A general theory of harmonic wave propagation in linear periodic systems with multiple coupling. JSV, 27(2):235-260, 1973.

[9] K. Mohan and M. Fazel. Iterative reweighted algorithms for matrix rank minimization. Journal of Machine Learning Research, 13(Nov):3441-3473, 2012.

[10] J. H. Park, P. S. Ma, and Y. Y. Kim. Design of phononic crystals for self-collimation of elastic waves using topology optimization method. Structural and Multidisciplinary Optimization, 51(6):1199-1209, 2015.

[11] B. Recht, M. Fazel, and P. A. Parrilo. Guaranteed minimum-rank solutions of linear matrix equations via nuclear norm minimization. SIAM review, 52(3):471-501, 2010.

[12] L. Vandenberghe and S. Boyd. Semidefinite programming. SIAM review, 38:49-95, 1996.

[13] G. Yi and B. D. Youn. A comprehensive survey on topology optimization of phononic crystals. Structural and Multidisciplinary Optimization, 54(5):1315-1344, 2016. 